\title{
Modulation of PI3K signaling to improve CAR T cell function
}

\author{
Wenting Zheng, Lindsay L. Jones and Terrence L. Geiger
}

During $\mathrm{CD}^{+} \mathrm{T}$ cell activation, engagement of the $\mathrm{T}$ cell receptor (TCR) along with costimulatory receptors triggers signaling pathways that lead to $\mathrm{T}$ cell expansion and differentiation. Among these, activation of phosphoinositide 3-kinase (PI3K) has a critical effect on $\mathrm{T}$ cell proliferation, survival, migration, and effector/ memory subset formation. Class I PI3Ks are composed of one of three isoforms of the p110 catalytic subunit (p110 $\alpha, \mathrm{p} 110 \beta$, or $\mathrm{p} 110 \delta$ ), that constitutively associate with a p85 regulatory subunit. Class I PI3Ks catalyze the phosphorylation of phosphatidylinositol 4,5-bisphosphate, generating phosphatidylinositol $(3,4,5)$-trisphosphate, which recruits proteins containing pleckstrin homology (PH) domains to the plasma membrane. PH domaincontaining targets, including AKT, initiate signaling and activate downstream effectors of cellular differentiation and metabolism [1]. During T cell activation, signaling through the $\mathrm{T}$ cell receptor, costimulatory molecules, and IL-2 receptor can all activate $\mathrm{PI} 3 \mathrm{~K} \delta$. TCR ligation induces zeta-chain associated ZAP70-mediated phosphorylation of LAT, which is required for the recruitment of PI3K to the membrane [2]. The costimulatory molecules CD28 and ICOS contain the consensus YxxM PI3K binding motif in their cytoplasmic tails. The mechanism of $\mathrm{PI} 3 \mathrm{~K} \delta$ activation through IL-2 signaling may involve LCK/FYN activity and control of the accumulation of phosphatidylinositol (3,4,5)-trisphosphate [3]. PI3K $\delta$ signaling after $\mathrm{T}$ cell activation leads to AKT-dependent inactivation and nuclear exclusion of FOXO1, which has been implicated in the downregulation of memory $\mathrm{T}$ cell markers such as IL-7R $\alpha$ and CD62L. PI3K $\delta$ also promotes mTOR signaling, leading to increased $\mathrm{T}$ cell metabolic activity which facilitates effector $\mathrm{T}$ cell differentiation and function [2].

While loss of PI3K activity is detrimental to immune function, constitutive activation of PI3K also impairs immunity because it preferentially promotes formation of short-lived terminally differentiated effector $\mathrm{T}$ cells at the expense of long lived memory $\mathrm{T}$ cells. Control of $\mathrm{T}$ cell activation and differentiation by PI3K is particularly relevant to Chimeric Antigen Receptor (CAR) T cell immunotherapy. CARs retarget genetically modified $\mathrm{T}$ lymphocytes through hybrid receptors that incorporate a tumor antigen-specific $\mathrm{scFv}$, one or more costimulatory domains (most commonly 41BB or CD28), and the CD3-zeta domain. CAR-T cells have experienced a surge in interest due to the now proven effectiveness of CD19-specific CAR-T cells in the treatment of precursor
B cell malignancies. CAR-modified T cells are not merely retargeted conventional $\mathrm{T}$ lymphocytes. The presence of a CAR on a T cell's surface alters its activation and differentiation, even in the absence of a complementary ligand. Constitutive self-signaling through CAR, related to both the $\mathrm{scFv}$ framework and the signaling domains, can lead to aberrant $\mathrm{T}$ cell behavior, including altered differentiation and decreased survival. This is significant as the effectiveness of CAR-T cells in patients is directly associated with their in vivo longevity. Long et. al. demonstrated that the presence of the CD28 costimulatory domain increased CAR-T cell exhaustion induced by persistent CAR self-signaling; the 4-1BB costimulatory domain had a lesser effect [4]. Using a panel of mutant CAR, our group identified a dominant role of the CAR CD3-zeta ITAMs in self-signaling. CD3-zeta significantly enhanced the constitutive activation of the PI3K, AKT, mTOR, and glycolysis pathways, and fostered formation of short-lived effector cells over central/stem memory cells [5].

Manipulation of PI3K signaling can be used to prevent altered CAR-T cell differentiation due to constitutive CAR self-signaling and foster longlived memory $\mathrm{T}$ cell development. We demonstrated that pharmacologic blockade of PI3K during CAR-T

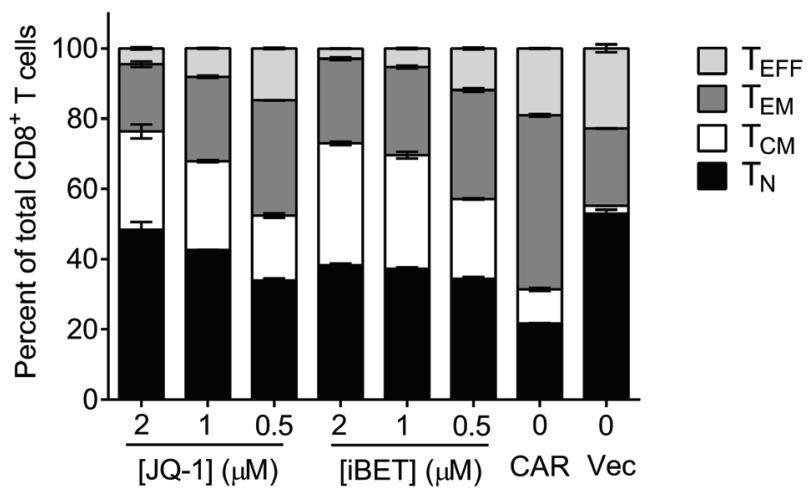

Figure 1: c-Myc inhibition restrains aberrant CAR-T cell differentiation ex vivo. $\mathrm{T}$ cells were activated with anti-CD3/anti-CD28 and transduced with CD33 CAR or empty vector. Five days after initial activation, CD33 CAR-T cells were treated with c-Myc inhibitors JQ-1 or iBET as indicated for four days. Percentages of $\mathrm{T}_{\mathrm{N}}$ (naïve; $\mathrm{CCR} 7^{+} \mathrm{CD} 45 \mathrm{RA} \mathrm{A}^{+}$), $\mathrm{T}_{\mathrm{CM}}$ (central memory; $\mathrm{CCR} 7^{+} \mathrm{CD} 45 \mathrm{RA}^{-}$), $\mathrm{T}_{\mathrm{EM}}$ (effector memory; $\mathrm{CCR}^{-} \mathrm{CD}^{-} 5 \mathrm{RA}^{-}$) and $\mathrm{T}_{\mathrm{EFF}}$ (effector; $\mathrm{CCR}^{-} \mathrm{CD}^{-} 5 \mathrm{RA}^{+}$) subsets of $\mathrm{CD}^{+} \mathrm{T}$ cells were determined by flow cytometry. Results indicate that the bromodomain inhibitors increase the proportion of longer-lived naïve and central memory phenotype $\mathrm{T}$ cells. 
manufacture and ex vivo expansion abrogated preferential effector $\mathrm{T}$ cell development and restored the CAR-T effector/memory ratio to that observed in empty vector transduced T cells. This improved in vivo T cell persistence and therapeutic activity in an AML model. Inhibition of p110 $\delta$ PI3K has also been found to enhance efficacy and memory in tumor-specific therapeutic CD8 T cells, while inhibition of $\mathrm{p} 110 \alpha \mathrm{PI} 3 \mathrm{~K}$ increased cytokine production and antitumor response [6, 7].

Downstream targets of PI3K include AKT, mTOR, and FOXO1, and are important in determining $\mathrm{CD}^{+} \mathrm{T}$ cell fate. Sustained AKT activation leads to T cell terminal differentiation. Its inhibition in CAR modified $\mathrm{T}$ cells results in an early memory phenotype and improved antitumor efficacy [8]. In our study, pharmacologic inhibition of AKT, mTOR, or glycolysis during ex vivo expansion of CAR-T cells promoted memory over effector cell formation. However, inhibition of these pathways also reduced CAR-T cell proliferative capacity, limiting therapeutic cell expansion [5]. These targets may therefore be suboptimal for inhibiting terminal effector differentiation, and will require further evaluation. $\mathrm{PI} 3 \mathrm{~K} / \mathrm{AKT}$ signaling can also promote c-myc activity by inhibiting c-myc phosphorylation by GSK-3 $\beta$ and its subsequent proteosomal degradation. Inhibition of c-myc by BET bromodomain inhibitors resulted in expansion of CD62 $\mathrm{L}^{+} \mathrm{CCR}^{+} \mathrm{T}$ cells with $\mathrm{T}_{\mathrm{N}}$ and $\mathrm{T}_{\mathrm{CM}}$ phenotypes, and adoptive transfer of inhibitor-treated CAR $\mathrm{T}$ cells extended survival in an ALL model [9]. We have also observed similar preservation of naïve and memory over effector phenotype in AML-specific CAR-T cells after treatment with BET bromodomain inhibitors (Figure 1). Recently, Singh et. al. found that the B cell adaptor for PI3-kinase (BCAP) is an important regulator of $\mathrm{CD}^{+}$ effector and memory $\mathrm{T}$ cell differentiation, highlighting yet another potential target in the PI3K pathway to balance effector and long-lived memory $\mathrm{T}$ cell generation [10]. It remains to be determined whether this or other downstream targets of PI3K will be effective in supporting therapeutic T cell survival and potency. Clearly, multiple studies now indicate that modulation of PI3K and its downstream targets is a promising approach to improve CAR-T cell efficacy by limiting CAR self-signaling effects and improving $\mathrm{T}$ cell memory formation, survival, and function. Optimizing the use of inhibitors of these pathways for clinical application is the next challenge.
Terrence L. Geiger: Department of Pathology, St. Jude Children's Research Hospital, Memphis, TN, USA

Correspondence to: Terrence L. Geiger, email terrence.geiger@stjude.org

Keywords: immunotherapy; CAR-T cell; PI3K; T cell receptor; cancer

Received: October 22, 2018

Published: November 09, 2018

\section{REFERENCES}

1. Kim EH, et al. Front Immunol. 2013; 4:20. https://doi.org/10.3389/fimmu.2013.00020.

2. Lucas CL, et al. Nat Rev Immunol. 2016; 16:702-14. https://doi.org/10.1038/nri.2016.93.

3. Ross SH, et al. Annu Rev Immunol. 2018; 36:411-33. https://doi.org/10.1146/annurev-immunol-042617-053352.

4. Long AH, et al. Nat Med. 2015; 21:581-90. https://doi.org/10.1038/nm.3838.

5. Zheng W, et al. Leukemia. 2018; 32:1157-67. https://doi.org/10.1038/s41375-017-0008-6.

6. Aragoneses-Fenoll L, et al. Front Immunol. 2018; 9:332. https://doi.org/10.3389/fimmu.2018.00332.

7. Abu Eid R, et al. Cancer Res. 2017; 77:4135-45. https://doi.org/10.1158/0008-5472.CAN-16-1925.

8. Klebanoff CA, et al. JCI Insight. 2017; 2. https://doi.org/10.1172/jci.insight.95103.

9. Kagoya Y, et al. J Clin Invest. 2016; 126:3479-94. https://doi.org/10.1172/JCI86437.

10. Singh MD, et al. J Exp Med. 2018; 215:2429-43. https://doi.org/10.1084/jem.20171820.

Copyright: Zheng et al. This is an open-access article distributed under the terms of the Creative Commons Attribution License 3.0 (CC BY 3.0), which permits unrestricted use, distribution, and reproduction in any medium, provided the original author and source are credited. 